\title{
ADAPTACIÓN Y EFICIENCIA AGRONÓMICA EN EL MAÍZ AMARILLO DURO (ZEA MAYS L.) EN DIFERENTES LOCALIDADES DE LA COSTA CENTRAL Y NORTE DEL PERÚ
}

\author{
ADAPTATION AND AGRONOMIC \\ EFFICIENCY IN HARD YELLOW CORN \\ (ZEA MAYS L.) IN DIFFERENT \\ LOCALITIES OF THE \\ CENTRAL AND NORTHERN COAST \\ OF PERU
}

\section{Lázaro Evaristo Lizarbe Alache ${ }^{1}$}

\section{Vega Córdova Eduardo Aníbal²}

\section{Lizarbe Córdova Jesús Armando ${ }^{3}$}

Universidad Nacional San Luis Gonzaga, Perú

\section{RESUMEN}

En la costa central y norte del Perú se llevó a $1 \quad$ Magister en Producción Agricola, Universidad Nacional "San Luis Gonzaga" lazarolizarbe16@hotmail.com https://orcid.org/0000-0002-8354-6361

2 Ingeniero Mecánico Eléctrico, Bachiller en Ingeniería Civil. vegcored_92@hotmail.com https://orcid.org/0000-0002-5341-5870, SIGELEC S.A.C. 3 Bachiller en Ingeniería Civil. jwske10@gmail.com Provias Descentralizado. https://orcid.org/0000-0002-6029-9716 cabo un Experimento en cada Valle, estudiándose Híbridos de Maíz Amarillo Duro XB-8014, XB8010, DK-7088, XB-8018, AG-1596, XB-8016, $X B-8030$. El suelo en cada Valle es de textura franca y franco arenosa, y el clima es apropiado para el cultivo. Diseño utilizado de bloques 
completamente randomizado, para los valles y factorial para el combinado valle (V) por híbrido (H).Efectuada las evaluaciones, se obtuvo los siguientes resultados:

\section{Rendimiento Maíz Grano Tn/há por Valles, destacan los Híbridos:}

Ica: DK-7088 con 14.923 Tn/há XB-8016 con 14.900 Tn/há, Cañete: XB-8016 con 12.495 Tn/há; AG-1596 con 12.374 Tn/há., Barranca: DK-7088 con $17.073 \mathrm{Tn} /$ há, XB-8018 con $15.005 \mathrm{Tn} /$ há, Moche : XB-8016 con 12.820 Tn/há, DK-7088 con $12.654 \mathrm{Tn} /$ há, Ferreñafe: DK-7098 con $10.136 \mathrm{Tn} /$ há; AG-15-.96 con 9.714 Tn/há, Motupe: XB-8018 con 14.500 Tn/há, XB-80.14 con 14.345 Tn/há.

\section{Rendimiento Maíz Grano Tn/há destacan:}

Para Combinado Valle (V) por Híbrido (H) = Barranca DK-7088 con 17.00 Tn/há, Barranca XB-8018 con 15.00 Tn/há, Ica por DK-7088 con $14.920 \mathrm{Tn} /$ há.

Valles $(\mathrm{V})=$ Barranca con $14.250 \mathrm{Tn} / \mathrm{há}$. Ica con $13.820 \mathrm{Tn} / \mathrm{há}$.

Híbridos $(H)=D K-7088$ con $13.420 \mathrm{Tn} /$ há; XB-8018 con 12.670 Tn/há; XB-8016 con $12.460 \mathrm{Tn} /$ há.

\section{PALABRAS CLAVE}

Híbridos de maíz, randomizado, factorial, valles, bloques.

\section{ABSTRACT}

In the central and northern coast of Peru, an Experiment was carried out in each Valley, studying Hard Yellow Corn Hybrids XB-8014, XB8010, DK-7088, XB-8018, AG-1596, XB-8016, XB -8030 The soil in each Valley has a loamy and sandy loam texture, the climate is appropriate for cultivation. Completely randomized block design used for the valleys and factorial for the combined valley $(\mathrm{V})$ by hybrid $(\mathrm{H})$. Once the evaluations were carried out, the following results were obtained:
Corn Grain Yield Tn / ha by Valleys, the Hybrids stand out:

Ica: DK-7088 with 14,923 Tn / ha, XB-8016 with 14,900 Tn / ha, Cañete: XB-8016 with 12,495 Tn / ha; AG-1596 with 12,374 Tn / ha., Barranca: DK7088 with 17,073 Tn / ha, XB-8018 with 15,005 Tn / ha, Moche: XB-8016 with 12,820 Tn / ha, DK7088 with 12,654 Tn / ha, Ferreñafe: DK-7098 with 10,136 Tn / ha; AG-15-.96 with 9,714 Tn / ha, Motupe: XB-8018 with 14,500 Tn / ha, XB-80.14 with 14,345 Tn / ha.

Corn Grain Yield Tn / ha include:

- For Combined Valley (V) by Hybrid $(H)=$ Barranca DK-7088 with 17.00 Tn / ha, Barranca XB-8018 with $15.00 \mathrm{Tn} /$ ha, Ica by DK-7088 with 14.920 Tn / ha.

- Valles (V) = Barranca with 14,250 Tn / ha. Ica with 13,820 Tn / ha.

- Hybrids (H) = DK-7088 with 13,420 Tn / ha; XB8018 with $12,670 \mathrm{Tn} / \mathrm{ha}$ XB-8016 with 12,460 $\mathrm{Tn} / \mathrm{ha}$.

Key words

Corn hybrids, randomized, factorial, valleys, blocks

\section{INTRODUCCIÓN}

El Cultivo de Maíz (Zea mayz L.), en especial el Amarillo Duro, es fuente principal para la alimentación de aves en la crianza de pollos y otras especies animales como materia prima para la industria, y de gran importancia económica, destinándose considerables áreas para la siembra y producción de granos de Maíz en seco.

Las regiones más importantes son Ica, Cañete (Sur Chico), Huacho (Norte Chico), Moche Chepen (Norte) de la Costa, peruana, valles con excelentes características maiceras, en especial los buenos Rendimientos, razón por lo que se efectúa el presente estudio, para determinar el lugar, valle, zona de mayor Rendimiento y cuál 
de los Híbridos nuevos se adaptaron al referido lugar o valle costero.

Como atractivo de este cultivo y la necesidad del Maíz, se tiene en el país un gran número de empresas nacionales y transnacionales que apuntan en proporcionar la mejor de su línea o híbrido de Maíz con buenos Rendimientos.

\section{SITUACIÓN PROBLEMÁTICA}

Según Alcatara y Quispe (2017), “La producción nacional de maíz tiene ciertas vulnerabilidades para abastecer la demanda nacional, como consecuencia de esto menciona una tendencia positiva en las importaciones peruanas de maíz". La tendencia de importación de MAD (Maíz amarillo durol es elevada debido a que existe una fuerte demanda por parte del sector avícola y porcino, quienes usan este grano como insumo para la producción de carne de ave y puerco. A su vez el consumo nacional de carne de ave y porcina aumentan año a año (Posada,2018).

El maíz (Zea mays L.) originario de América, presenta uno de los aportes más valiosos a la seguridad alimentaria mundial. Junto con el arroz y el trigo son considerados como las tres poáceas más cultivadas en el mundo. Asimismo, en el transcurso del tiempo, diversas instituciones mundiales, estatales y privadas vienen realizando estudios con el objetivo principal de incrementar el rendimiento y la producción con nuevos híbridos resistentes a factores bióticos y abióticos (INIA, 2015).

MINAGRI (2019), informa a través de la Serie de Estadísticas de Producción Agrícola (SEPA) el área cosechada de maíz chala a nivel nacional para los años 2015, 2016 y 2017 fueron de 35 336 ha, 34885 ha y 36311 ha respectivamente. Los rendimientos promedios nacionales para los años 2015, 2016 y 2017 fueron de $46980 \mathrm{~kg} / \mathrm{ha}$, $46836 \mathrm{~kg} / \mathrm{ha}$ y $49101 \mathrm{~kg} / \mathrm{h}$ a respectivamente. A lo largo de estos años, se puede apreciar que los departamentos con mayores áreas cosechadas y rendimientos promedios regionales son Arequipa, La Libertad y Lima.

\section{MARCO TEÓRICO}

\subsection{Antecedentes}

Vásquez 2019, El experimento se condujo bajo un diseño de bloques completos al azar con arreglo factorial, 3Vx2D con 5 repeticiones. Se encontró interacción entre Densidades de siembra por Variedades en las variables diámetro de tallo, peso fresco de hojas, peso fresco por planta, peso seco de mazorca y rendimiento de materia seca. El hibrido EXP-05 con la densidad de siembra de 111111 plantas/hectárea mostro una mejor adaptabilidad forrajera para rendimiento de forraje verde y materia seca. En el rendimiento de forraje verde, todos los tratamientos superaron las 80 toneladas por hectárea; la mejor densidad fue la de 111111 plantas/ha con un promedio de $100 \mathrm{t} / \mathrm{ha}$, y la mejor variedad EXP-05 con un promedio de $103.97 \mathrm{t} / \mathrm{ha}$. En el rendimiento de materia seca por hectárea, todos los tratamientos superaron las 26 toneladas por hectárea. El acame en campo, están directamente relacionados con la altura de planta $(\mathrm{cm})$ y la altura de mazorca (cm) principalmente con la densidad de 111111 plantas/ha en donde el mayor incremento se tuvo con PM-213.

Castope (2014), estudio 26 diferentes variedades comerciales y experimentales de maíz amarillo duro con fines forrajeros (EXP-01, EXP-03, EXP05, PM-213, etc.). Las variedades PM-213 y la EXP-05, obtuvieron rendimientos de materia fresca de entre 65 a $58 \mathrm{t} /$ ha y entre un 25 a $30 \%$ de materia seca como rendimiento por hectárea. Los híbridos comerciales presentaron alturas promedio de $3.03 \mathrm{~cm}$ y $2.57 \mathrm{~cm}$ además que todos presentaron un promedio de 2 centímetros para el valor del diámetro de tallo. La floración masculina y femenina oscilo entre los 72 a74.5 días respectivamente. Como conclusión final, menciona que las variedades experimentales 
(EXP's) y la variedad PM-213 mostraron un mejor comportamiento para fines forrajeros.

Banda (2019) El objetivo general de la investigación fue determinar el potencial de rendimiento de los 3 híbridos promisorios y 5 testigos (híbridos comerciales) de MAD, empleando una investigación de nivel explicativo y diseño experimental. Se utilizó un DBCA con cuatro repeticiones y ocho tratamientos (tres híbridos promisorios de MAD desarrollados por el INIA "Vista Florida", Ferreñafe y cinco híbridos comerciales de MAD empleados en la región Lambayeque). Según los resultados, el comportamiento agronómico de los híbridos promisorios PMAD - 1 y PMAD - 2 fue estadísticamente igual al de los híbridos comerciales. También, se evidenció que, el comportamiento agronómico del híbrido promisorio PMAD - 3 fue estadísticamente inferior a los híbridos promisorios PMAD 1. PMAD - 2 y los híbridos comerciales del experimento (INIA 619 Megahíbrido, DK - 7088, AGRHICOL XB - 8010, DOW 2B688 e INSIGNIA 860). Finalmente, se recomienda el uso de los híbridos promisorios PMAD - 1, PMAD - 2 para el desarrollo comercial de semilla híbrida mejorada de MAD.

Quimi (2015), desarrolló una investigación cuyo objetivo fue determinar el comportamiento, adaptabilidad y manejo de híbridos triples promisorios de maíz que conlleven a mejorar la rentabilidad de los productores. En la cuales sus resultados en cuanto a rendimiento por hectárea mostraron que el híbrido experimental (SM45x SV15) obtuvo el mayor rendimiento con $7428,73 \mathrm{~kg} . \mathrm{h}-1$ siendo estadísticamente superior a los demás materiales evaluados. Y el hibrido experimental (SM45 x SV35) SV39 obtuvo menor rendimiento con $6199,32 \mathrm{~kg} . \mathrm{h}-1$, y en cuanto a los materiales comerciales, el que más sobresalió fue el híbrido AG - 003 con 6780,56 kg.h -1 .
Armijos y Ruilova (2014), en su investigación tuvieron como objetivo evaluar el comportamiento agronómico y adaptación de 12 híbridos comerciales de maíz (Zea mays L.) y 4 híbridos experimentales en los cantones Zapotillo y Pindal provincia de Loja y el cantón Santa Elena. En las cuales sus resultados muestran que los materiales comerciales mostraron mejor comportamiento agronómico y adaptación en Zapotillo, Pindal y Santa Elena en el que destacaron el híbrido Dekalb 7088 con rendimiento de 17 12,64 t/ha; Triunfo con 10,92 t/ha e INIAP H-602 con rendimiento de $10,26 \mathrm{t} / \mathrm{ha}$. $Y$ en híbridos experimentales reporta rendimientos de 7,$58 ; 7,21 ; 7,12 ; 7,02$ y $6,72 \mathrm{t} / \mathrm{ha}$.

\subsection{Base Teórica.}

El maíz en el nuevo mundo es considerado el principal cereal domesticado y fue la base alimenticia de las civilizaciones maya, azteca e inca. Las teorías genéticas sobre el origen del maíz son muy diversas, pero se originó como planta cultivada en algún lugar de América humano en sus diversas formas como para su utilización forrajera. Gracias a su capacidad de adaptación, su elevado rendimiento y las posibilidades futuras de mejoras por la vía genética.

\subsection{Clasificación botánica.}

Reino : Plantae, División : Magnoliophyta, Clase : Liliopsida, Subclase : Commelinidae, Orden : Poales, Familia : Poaceae, Género : Zea Especie : Mays

Nombre Científico : Zea mays L.

\subsection{Descripción botánica:}

El maíz es una planta anual de gran desarrollo vegetativo, que puede alcanzar hasta 3.5 metros de altura (lo normal es de 2 a 2.5 metros) 
Raíz: todo el sistema radical de la planta adulta es adventicio.

Tallo: el tallo central del maíz es un eje formado por nudos y entrenudos, cuyo número y longitud varían notablemente. La parte inferior y subterránea del tallo tiene entrenudos muy cortos de los que salen las raíces principales y los brotes laterales. Los entrenudos superiores son cilíndricos, en corte transversal se observa que la epidermis se forma de paredes gruesas y haces vasculares cuya función principal es la conducción de agua y substancias nutritivas obtenidas del suelo o elaboradas en las hojas.

Hoja: este cereal tiene la hoja similar a la de otras poáceas; está constituida de vaina, cuello y lámina. La lámina es una banda angosta y delgada hasta de $1.5 \mathrm{~m}$ de largo por $0.1 \mathrm{~m}$ de ancho, que termina en un ápice muy agudo. El nervio central está bastante desarrollado, es prominente en el envés de la hoja y cóncavo en el lado superior.

Mazorca: al contrario de la mayor parte de las poáceas, en el maíz la espiga es compacta y está protegida por las hojas transformadas, que en la mayoría de los casos la cubren por completo

Panoja: se encuentra localizada en la parte terminal del tallo, formada por un eje principal y que es la prolongación del tallo y termina en la borla, presentando ramas primarias, secundarias y terciarias (Espinoza 2016).

\subsection{Rendimiento del maíz amarillo duro.}

Los componentes del rendimiento del maíz están determinados por características biométricas de la mazorca llongitud y diámetro de la mazorca, número de hileras y número de granos por hilera), número de mazorcas por planta, peso de 1000 granos, etc. Diversos estudios así lo corroboran, sobre todo los realizados por Ferraris y Couretot citado por Chura J 2014 ), quienes evaluaron el comportamiento de 26 híbridos de maíz y los siguientes componentes del rendimiento: número de granos $/ \mathrm{m}^{2}$, peso de 1000 granos y número de hileras por mazorca.l Rivetti citado por Chura J. 2014)luego de evaluar el rendimiento de grano y sus componentes bajo diferentes regímenes de riego, estableció los siguientes componentes del rendimiento: número de hileras por mazorca, número de granos $/ \mathrm{m}^{2}$, número de granos por hilera y el peso de 1000 granos. Características deseables en plantas de maíz son, principalmente, poca altura de planta, mayor precocidad y hojas superiores erectas lángulo de inserción de hoja pequeño). En la costa peruana, por ejemplo, el sistema de producción requiere híbridos de maíz más precoces y de porte más bajo, que puedan sembrarse a altas densidades. Asimismo, hojas superiores erectas combinadas con hojas horizontales inferiores dan lugar a un uso más eficiente de la radiación por parte de la capa total de hojas (Paliwal, citado por Chura J. 2014).

Rendimiento. Es la consideración fundamental en la producción del maíz híbrido, también atribuye que ello, es el objetivo más concreto con que trabaja el mejorador del maíz, básicamente está determinado por la acción de numerosos genes, muchos de los cuales afectan a procesos vitales de la planta, como la nutrición, la fotosíntesis, la 26 transpiración, la translocación y el almacenamiento de los principios nutritivos. También afectan directa o indirectamente al rendimiento, la precocidad, la resistencia a los insectos y enfermedades y otras características que pueden evaluarse con mayor precisión que el rendimiento por selección visual, por lo que generalmente se utiliza como base la selección visual en la obtención de líneas autofecundadas. Diaz S. (2019)

Maiz Híbrido. “Técnicamente un híbrido es la primera generación F1 de un cruzamiento entre dos genotipos claramente diferentes. Normalmente se producen numerosos tipos de híbridos en todos los programas de mejoramiento para combinar diferentes caracteres de los 
distintos genotipos" El vigor híbrido es debido a dos posibles hipótesis: al dominancia, que en base a la relación entre recesividad, aparición o incremento de los genes recesivos en el individuo y los efectos detrimentales; explica el incremento debido al vigor híbrido por la presencia de la heterocigosidad que encubre los efectos detrimentales de los genes recesivos por los dominantes, de efectos favorables; $y$, b) sobredominancia, la cual asume que el heterocigoto (híbrido) es superior a cualquiera de los dos homocigotos (líneas endocríadas)

Obtención de maíces híbridos. Una cruza simple, $A \times B$, se hace combinando dos líneas puras. Las cruzas simples tienden a ser de rendimiento ligeramente mayor y más uniformes en las características de la planta y la mazorca que otros tipos de híbridos. Estos híbridos pueden formarse mediante polinización manual o cultivando dos variedades en bloques alternados en una parcela aislada y desespigando (emasculando), antes de que hayan esparcido polen, todas las plantas de la variedad en la que se va a producir semilla. Diaz S. (2019)

\section{METODOLOGÍA}

De los Materiales y Métodos

a) Ubicación de los Campos Experimentales El estudio se realizó en 6 Diferentes ciudades del país.

- Ica (Provincia y Departamento de Ica)

- Cañete (Provincia, Cañete y Departamento de Limal

- Barranca (Provincia Barranca, Departamento de Limal

- Moche (Provincia Trujillo. Departamento La Libertad).

- Ferreñafe: (Provincia. Ferreñafe, Departamento de Lambayeque)

- Motupe (Provincia Chiclayo y Departamento de Lambayequel

En cada de ellas el área Experimental es el mismo; los Tratamientos (híbridos) y las repeticiones o Block tienen idénticas Fuentes de Variación (F.V)

b) Historia de los Campos. La mayoría de los campos donde se efectúan los Experimentos pertenece a agricultores de avanzada, maiceros de experiencia en la siembra de Maíz Amarillo Duro.

Todos los campos son regados con agua de subsuelo bajo el sistema de gravedad (Agua de pozol.

c) Análisis de Suelo.En informes Nacionales referente al Análisis de suelos se indica que los campos costeños son preferentemente de Textura Franco Arenoso, suelos bastantes profundos, retentivos, de $\mathrm{pH}$ alcalinos, deficiente en Nitrógeno y Materia Orgánica, medio en Fósforo y alto en Potasio disponible.

d) Observaciones MeteorológicasDatos meteorológicos tomados por SENAMHI Indican que el clima a nivel de los Valles han variado, en especial la Temperatura que ha cambiado sustancialmente en la campaña 2013-2014, ocurriendo lo mismo con las Horas de Sol diaria y mensual, la Humedad Relativa (\%) fue más baja que en campañas anteriores, sin embargo estos han sido adecuados para el crecimiento y desarrollo del cultivo.

e) Características de los Híbridos en estudio. Se evaluaron los híbridos

- XB - 8018, XB-8014, XB-8016, XB-8010, DX-7888 (DELKALB), XB-8030

AG-1596

Cómo característica principal:

- Maíz de Grano Amarillo Duro

- Tamaño Mazorca Grande

- $\quad$ Altura de Planta 2.00-2.30m

- Posesión de las Hojas es Erecta

- $\quad$ Potencial de Rendimiento - excelente

16,000 a 8,000 kg de granos seco / hectárea.

f) Tratamientos empleados

Se probaron 5 (cinco) Híbridos de Maíz con progenitores en clave y que tienen de origen a Diferentes países. 
Tal como se indicó en la ubicación del Experimento, los mismos Tratamientos se evaluaron en 6 Valles de la Costa del Sur Chico y Costa del Norte Chico (Ica, Cañete, Barranca, Moche, Ferreñafe y Motupe), aplicándose el mismo Diseño Experimental para cada valle y posteriormente, se efectúa el análisis Combinado.

g) Diseño Experimental. Se empleó el Diseño en Bloques Completamente Randomizado, presentando 5 Tratamientos (5 Híbridos de Avanzada y 2 Híbridos testigo), conducidos en 4 Bloques o Repeticiones, teniéndose 28 Unidades Experimentales con las siguientes características.

* Del ANVA (Análisis de Variancia)

\begin{tabular}{|l|c|}
\hline $\begin{array}{c}\text { F.V. (Fuente de } \\
\text { Variación) }\end{array}$ & G.L. (Grado de Libertad) \\
\hline Total & 27 \\
Bloques & 3 \\
Tratamientos & 6 \\
EE (Error & 18 \\
Experimental) & \\
\hline
\end{tabular}

- Del Modelo Matemático

$x_{i j}=\mu+\alpha_{i}+\beta_{j}+\varepsilon_{i j}$

\section{Donde:}

$x_{i j}=$ Cualquier observación

$$
\begin{aligned}
& \boldsymbol{\mu}=\text { Media de la Población, } \\
& \boldsymbol{\alpha}_{i}=\text { Efecto aditivo del iesimo tratamiento }
\end{aligned}
$$

, $\boldsymbol{\beta}_{j} \boldsymbol{\beta}_{j}=$ Efecto aditivo del jota ésimo Boque o Repetición

$\varepsilon_{i j}=$ Variable al Azar que significa el Error a que

$\varepsilon_{i j}=$ Variable al Azar que significa el Error a que está sujeta a cada observacion

\subsection{De los instrumentos de investigación}

Durante el estudio Experimental sobre el crecimiento y desarrollo de las Variedades de Maíz Amarillo Duro, se emplearon los siguientes instrumentos logísticos:

De campo y de Oficina.

\subsection{Contrastación de Hipótesis}

La Hipótesis planteada sobre la agricultora moderna del Maíz Amarillo Duro estadísticamente es superior a los maíces criollos y mejorados producidos porDiferentes empresas demostrándose por los Rendimientos obtenidos que superan las 15 tn/há a comparación de los que están en el mercado que tienen potenciales de Rendimiento de 8 a $12 \mathrm{tn} /$ há. 


\section{RESULTADOS}

CUADRO $\mathrm{N}^{\circ} 01$

PRINCIPALES CARACTERÍSTICAS FENOLÓGICAS DEL EXPERIMENTO EN MAÍZ AMARILLO DURO EN LOS VALLES DE ICA, LIMA, LA LIBERTAD, LAMBAYEQUE / DIFERENTES HÍBRIDOS

\begin{tabular}{|c|c|c|c|c|c|c|c|c|c|c|c|}
\hline $\begin{array}{l}\text { CLAVE } \\
\text { LITER }\end{array}$ & $\begin{array}{l}\text { VALLES/ } \\
\text { HÍBRIDOS }\end{array}$ & $\begin{array}{l}\text { LONGITUD } \\
\text { DE } \\
\text { MAZORCA }\end{array}$ & $\begin{array}{c}\text { DIAMETRO } \\
\text { DE } \\
\text { MAZORCA }\end{array}$ & $\begin{array}{c}\text { NÚMERO } \\
\text { DE HILERAS } \\
\text { X MAZORCA }\end{array}$ & $\begin{array}{c}\text { NÚMERO } \\
\text { DE GRANOS } \\
\text { POR HILERA }\end{array}$ & $\begin{array}{c}\text { PESO } \\
\text { DE } \\
\text { MAZORCA }\end{array}$ & $\begin{array}{c}\text { PESO } \\
\text { DE } \\
\text { GRANO }\end{array}$ & $\begin{array}{l}\text { PESO DE } \\
\text { CORONTA }\end{array}$ & $\begin{array}{c}\% \\
\text { DE GRANO }\end{array}$ & $\begin{array}{c}\text { PESO } \\
\text { DE } 1000 \\
\text { GRANOS }\end{array}$ & $\begin{array}{c}\text { PESO X } \\
\text { PARCELA } \\
\text { CORREGIDO }\end{array}$ \\
\hline \multicolumn{2}{|l|}{ VALLE } & $(\mathrm{cm})$ & $(\mathrm{cm})$ & $\left(N^{0}\right)$ & $\left(N^{0}\right)$ & $(\mathrm{kg})$ & $(\mathrm{kg})$ & $(\mathrm{kg})$ & $(\%)$ & $(\mathrm{kg})$ & $(\mathrm{kg})$ \\
\hline$V_{1}$ & $\begin{array}{l}\text { Dpto. Ica. } \\
\text { Valle Ica - } \\
\text { Macacona }\end{array}$ & 16.8 & 5.17 & 16.30 & 34.70 & 9.662 & 8.212 & 1.587 & 84.07 & 0.33625 & 8.772 \\
\hline$V_{2}$ & $\begin{array}{l}\text { Dpto. Lima. } \\
\text { Valle Cañete- } \\
\text { Cerro Alegre }\end{array}$ & 15.65 & 4.75 & 13.85 & 34.00 & 8.475 & 7.125 & 1.320 & 84.94 & 0.36057 & 5.819 \\
\hline $\mathrm{V}_{3}$ & $\begin{array}{l}\text { Dpto. } \\
\text { Lima. Valle } \\
\text { Barranca - La } \\
\text { Paz }\end{array}$ & 15.74 & 4.84 & 14.40 & 33.30 & 10.800 & 9.125 & 1.650 & 84.47 & 0.35107 & 11.907 \\
\hline$V_{4}$ & $\begin{array}{l}\text { Dpto. La } \\
\text { Libertad. } \\
\text { Valle Trujillo - } \\
\text { Moche }\end{array}$ & 15.47 & 4.76 & 15.60 & 34.10 & 7.875 & 6.800 & 1.050 & 86.65 & 0.35035 & 7.348 \\
\hline $\mathrm{V}_{5}$ & $\begin{array}{l}\text { Dpto. } \\
\text { Lambayeque. } \\
\text { Valle Trujillo- } \\
\text { Ferreñafe }\end{array}$ & 14.98 & 4.4 & 15.20 & 32.05 & 6.575 & 5.550 & 1.037 & 84.15 & 0.30803 & 5.702 \\
\hline $\mathrm{V}_{0}$ & $\begin{array}{l}\text { Dpto. } \\
\text { Lambayeque. } \\
\text { Valle } \\
\text { Chiclayo. } \\
\text { Motupe }\end{array}$ & 15.75 & 4.86 & 15.10 & 34.00 & 9.100 & 7.650 & 1.425 & 85.20 & 0.35339 & 8.493 \\
\hline \multicolumn{12}{|c|}{ HÍBRIDOS } \\
\hline $\mathrm{H}$ & XB-8014 & 15.83 & 4.75 & 14.20 & 34.60 & 8.775 & 5.125 & 1.375 & 84.71 & 0.35795 & 8.016 \\
\hline $\mathrm{H}_{2}$ & XB-8010 & 16.61 & 5.08 & 14.20 & 33.10 & 7.500 & 6.175 & 1.237 & 85.11 & 0.33920 & 6.943 \\
\hline $\mathrm{H}_{3}$ & DK-7088 & 14.94 & 4.81 & 17.00 & 34.15 & 9.625 & 8.200 & 1.437 & 87.11 & 0.28925 & 8.571 \\
\hline $\mathrm{H}_{4}$ & XB-8018 & 15.66 & 5.00 & 15.00 & 32.35 & 8.750 & 7.350 & 1.500 & 83.46 & 0.34825 & 8188. \\
\hline $\mathrm{H}_{5}$ & AG-1596 & 15.53 & 5.00 & 17.10 & 33.35 & 8.612 & 7.362 & 1.275 & 85.81 & 0.33500 & 7.875 \\
\hline $\mathrm{H}_{0}$ & XB-8016 & 15.46 & 4.48 & 13.60 & 32.80 & 8.612 & 7.400 & 1.375 & 85.22 & 0.32014 & 7.972 \\
\hline $\mathrm{H}_{7}$ & XB-8030 & 15.72 & 4.77 & 13.65 & 32.80 & 8.400 & 6.950 & 1.400 & 83.22 & 0.30839 & 7.279 \\
\hline
\end{tabular}




\section{ANALISIS}

Las principales características fenológicas del cultivo de Maíz Amarillo Duro, que comprende el presente estudio, correspondiente a la ubicación en el Orden de Mérito en los Factores Valles (V) y los Híbridos(H) observándose que en Longitud de
Mazorca $(\mathrm{cm})$ el Valle de Ica - Macacona destaca con $16.18 \mathrm{~cm}$, de tamaño de Mazorca, sigue el Valle de Chiclayo - Motupe con $15.75 \mathrm{~cm}$, luego el Valle de Barranca - La Paz con 15.74 cm; en los Híbridos sobresale la Clave XB-8010 con $16.61 \mathrm{~cm}$, sigue la clave XB-8814 con $15.84 \mathrm{~cm}$, luego la clave XB-8030 con $15.72 \mathrm{~cm}$ de largo.

\section{CUADRO $\mathrm{N}^{\circ} 2$}

Análisis de variancia del rendimiento tn/há del experimento maíz amarillo duro

\begin{tabular}{|c|c|c|c|c|c|c|c|}
\hline F.V & SC & G.L & C.M & F. c & \multicolumn{2}{|c|}{ F.t } & SIGN \\
\hline Total & 1084.868 & 167 & - & - & - & - & ${ }^{-}$ \\
\hline Block & 206.385 & 3 & 68.795 & 61.369 & 2.68 & 3.94 & ${ }^{* *}$ \\
\hline Trat. O Combinado & 740.568 & 41 & 18.062 & 16.112 & 1.49 & 1.75 & ${ }^{* *}$ \\
\hline Valle (V) & 563.764 & 5 & 112.752 & 100.581 & 2.29 & 3.17 & ${ }^{* *}$ \\
\hline Híbrido (H) & 100.038 & 6 & 16.673 & 14.873 & 2.17 & 2.95 & ${ }^{* *}$ \\
\hline VH & 76.766 & 30 & 2.558 & 2.281 & 1.55 & 1.85 & ${ }^{* *}$ \\
\hline E.E & 137.915 & 123 & 1.121 & - & - & - & ${ }^{*}$ \\
\hline
\end{tabular}

$\bar{x}=12.18$

$S^{2}=1.121$

$$
S=1.058 \quad S_{x}=0.529
$$

$$
\begin{gathered}
S_{\bar{a}}=0.748 \\
C . V=8.68 \%
\end{gathered}
$$

\section{ANÁLISIS.}

Se presenta el Análisis de Variancia (ANVA) del Rendimiento Toneladas por Hectárea del Experimento de Maíz Amarillo Duro; en el Cuadro se observa que existe Alta Significación $(* *)$ para Combinado $(\mathrm{V} x \mathrm{H})$, Valle $(\mathrm{V})$, Híbridos(H) y la Interacción $(\mathrm{VH})$, lo que nos permite indicar que en Tratamientos o Combinados (VH) existen Diferencia Estadística Significativa, sucediendo lo mismo para Valle (V) e Híbridos de Maíz (H), con un Coeficiente de Variación de $8.68 \%$ considerado excelente para este tipo de investigación. 
CUADRO N³

Orden de mérito a la prueba de Duncan del rendimiento del experimento maíz amarillo duro

\begin{tabular}{|c|c|c|c|c|c|}
\hline \multicolumn{2}{|c|}{ Clave } & \multirow{2}{*}{$\begin{array}{c}\text { Combinados } \\
\text { (VxH) }\end{array}$} & \multirow[t]{2}{*}{$(\mathrm{Kg})$} & \multirow[t]{2}{*}{ DUNCAN } & \multirow[b]{2}{*}{ D.N. } \\
\hline Num & Liter & & & & \\
\hline \multicolumn{6}{|c|}{ COMBINADO VALL } \\
\hline 17 & $v_{3} h_{3}$ & Barranca x DK-7088 & 17.07 & $\mathrm{a}$ & $1^{0}$ \\
\hline 18 & $v_{3} h_{4}^{3}$ & Barranca x XB-8018 & 15.00 & $\mathrm{~b}$ & $2^{\circ}$ \\
\hline 3 & $v_{1} h_{3}$ & Tca x DK-7088 & 14.92 & $\mathrm{~b}$ & $2^{\circ}$ \\
\hline 6 & $v_{1} h_{6}$ & Ica x XB - 8016 & 14.90 & $\mathrm{~b}$ & $2^{\circ}$ \\
\hline 15 & $v_{3} h_{1}$ & Barranca x XB8014 & 14.65 & $\mathrm{~b}$ & $2^{\circ}$ \\
\hline 39 & $v_{6} h_{4}$ & Motupe x XB-8018 & 14.50 & $\mathrm{~b}$ & $2^{\circ}$ \\
\hline 36 & $\mathrm{v}_{6} \mathrm{~h}_{1}$ & Motupe x XB-8014 & 14.34 & $\mathrm{~b}$ & $2^{\circ}$ \\
\hline 19 & $v_{3} h_{5}$ & Barranca x AG-1596 & 14.07 & $\mathrm{~b}$ & $2^{\circ}$ \\
\hline 38 & $\mathrm{v}_{6} \mathrm{~h}_{3}$ & Motupe x DK-7088 & 14.01 & b c & $2^{\circ}$ \\
\hline 1 & $v_{1} h_{1}$ & Ica $\times$ XB-8014 & 13.79 & b c & $2^{\circ}$ \\
\hline 20 & $v_{3} h_{6}$ & Barranca x XB-8016 & 13.68 & b c & $2^{\circ}$ \\
\hline 5 & $v_{1}^{3} h_{5}^{6}$ & Ica x AG-1596 & 13.63 & b c & $2^{\circ}$ \\
\hline 40 & $v_{6} h_{5}$ & Motupe x AG-1596 & 13.53 & $\mathrm{C}$ & $3^{\circ}$ \\
\hline 21 & $\mathrm{v}_{3} \mathrm{~h}_{7}$ & Barranca x XB-8030 & 13.39 & $\mathrm{C}$ & $3^{\circ}$ \\
\hline 4 & $v_{3} h_{4}$ & Tca x XB-8018 & 13.22 & $\mathrm{C}$ & $3^{\circ}$ \\
\hline 2 & $v_{1} h_{2}$ & Ica $\times$ XB-8014 & 13.21 & C & $3^{\circ}$ \\
\hline 7 & $v_{1} h_{7}$ & Ica x XB-8030 & 13.08 & $\mathrm{C}$ & $3^{\circ}$ \\
\hline 41 & $v_{6} h_{6}$ & Motupe x XB-8016 & 13.00 & c & $3^{\circ}$ \\
\hline 27 & $\mathrm{v}, \mathrm{h}$ & Moche $x$ XB-8016 & 12.81 & C & $3^{\circ}$ \\
\hline 24 & $v_{4} h_{3}$ & Moche x DK-7088 & 12.65 & $\mathrm{C}$ & $3^{\circ}$ \\
\hline 11 & $v_{2} h_{4}^{3}$ & Cañete x XB-8018 & 12.49 & $\mathrm{C}$ & $3^{\circ}$ \\
\hline 12 & $v_{2} h_{5}$ & Cañete x AG-1596 & 12.37 & $\mathrm{C}$ & $3^{\circ}$ \\
\hline 25 & $v_{4} h_{4}$ & Moche x XB-8018 & 12.34 & $\mathrm{C}$ & $3^{\circ}$ \\
\hline 42 & $\mathrm{v}_{6} \mathrm{~h}_{7}$ & Motupe x XB-8030 & 12.21 & $c d$ & $3^{\circ}$ \\
\hline 8 & $v_{2} h_{1}$ & Cañete x XB-8014 & 12.16 & $c d$ & $3^{\circ}$ \\
\hline 16 & $\mathrm{v}_{3} \mathrm{~h}_{2}$ & Barranca x XB-8010 & 11.92 & $d$ & $4^{\circ}$ \\
\hline 10 & $v_{2} h_{3}$ & Cañete x DK-7088 & 11.77 & $d$ & $4^{\circ}$ \\
\hline 22 & $v_{4} h_{1}^{3}$ & Moche $x$ XB-8014 & 11.74 & $\mathrm{~d}$ & $4^{\circ}$ \\
\hline 13 & $v_{2} h_{4}$ & Cañete x XB-8018 & 11.39 & $\mathrm{~d}$ & $4^{\circ}$ \\
\hline 14 & $v_{2} h_{7}^{4}$ & Cañete x XB-8030 & 11.25 & d & $4^{\circ}$ \\
\hline 37 & $\mathrm{v}_{6} \mathrm{~h}_{2}$ & Motupe x XB-8010 & 11.25 & $d$ & $4^{\circ}$ \\
\hline 26 & $v_{4} h_{5}$ & Moche x AG-1596 & 10.49 & $\mathrm{~d} e$ & $4^{\circ}$ \\
\hline 9 & $\mathrm{v}_{2} \mathrm{~h}_{2}$ & Cañete x XB-8010 & 10.39 & $\mathrm{~d} e$ & $4^{\circ}$ \\
\hline 23 & $v_{4} h_{2}^{2}$ & Moche $\times$ XB-8010 & 10.24 & $d e$ & $4^{\circ}$ \\
\hline 31 & $v_{5} h_{3}^{2}$ & Ferreñafe x DK-7088 & 10.13 & $\mathrm{e}$ & $5^{\circ}$ \\
\hline 28 & $v_{4} h_{7}$ & Moche $x$ XB-8030 & 10.04 & $\mathrm{e}$ & $5^{\circ}$ \\
\hline 33 & $v_{5} h_{5}$ & Ferreñafe x AG-1596 & 9.70 & $\mathrm{e}$ & $5^{\circ}$ \\
\hline 34 & $v_{5} h_{6}$ & Ferreñafe $x$ XB-8016 & 8.97 & $e f$ & $5^{\circ}$ \\
\hline 32 & $v_{5} h_{4}$ & Ferreñafe x XB-8018 & 8.46 & $f$ & $6^{\circ}$ \\
\hline 29 & $v_{5} h_{1}^{4}$ & Ferreñafe x XB-8014 & 8.42 & $f$ & $6^{\circ}$ \\
\hline 30 & $v_{5} h_{2}$ & Ferreñafe x XB-8010 & 8.42 & $f$ & $6^{\circ}$ \\
\hline 35 & $\mathrm{v}_{5} \mathrm{~h}_{7}$ & Ferreñafe x XB-8030 & 8.22 & $f$ & $6^{\circ}$ \\
\hline \multicolumn{6}{|c|}{ FACTOR (VALLE (V) } \\
\hline 3 & $v_{3}$ & Barranca (Lima) & 14.25 & a & $1^{\circ}$ \\
\hline 1 & $v_{1}$ & Ica (Ica) & 13.82 & $\mathrm{a}$ & $1^{\circ}$ \\
\hline 6 & $v_{6}$ & Moche (Lambayeque) & 13.27 & $\mathrm{a} \quad \mathrm{b}$ & $1^{\circ}$ \\
\hline 4 & $\mathrm{~V}_{4}$ & Moche (La Libertad) & 11.47 & $\mathrm{~b}$ & $2^{\circ}$ \\
\hline 2 & $\mathrm{~V}_{0}$ & Cañete (Lima) & 11.40 & $\mathrm{~b}$ & $2^{\circ}$ \\
\hline 5 & $V_{5}^{2}$ & tons & 8.90 & $\mathrm{C}$ & $3^{\circ}$ \\
\hline 3 & $h_{3}$ & DK-7088 & 13.42 & $a$ & $1^{\circ}$ \\
\hline 4 & $\mathrm{~h}_{4}$ & XB-8018 & 12.67 & $a$ & $1^{\circ}$ \\
\hline 6 & $h_{6}$ & XB-8016 & 12.46 & $a$ & $1^{\circ}$ \\
\hline 5 & $h_{5}$ & AG-1596 & 12.30 & $a$ & $1^{\circ}$ \\
\hline 1 & $\frac{s}{h_{1}}$ & XB-8014 & 12.18 & $a b$ & $2^{\circ}$ \\
\hline 7 & $h_{7}$ & XB-8030 & 11.37 & $\mathrm{~b}$ & $2^{\circ}$ \\
\hline 2 & $h_{2}$ & XB-8010 & 10.90 & $\mathrm{~b}$ & $2^{\circ}$ \\
\hline
\end{tabular}




\section{ANALISIS}

Sobre el Orden de Mérito a la Prueba de Amplitudes Estudiantizadas Significativas

de DUNCAN $\propto 0.05 \propto 0.05$ del Rendimiento del Experimento de Maíz Amarillo Duro, en Diferentes valles de la Costa Peruana llca, Cañete, Barranca; Moche, Ferreñafe, Motupel y los Híbridos de Avanzada (XB-8014; XB-8010; DK-7088; XB-8018, AG-1596; XB-8016; XB8030) e identificar al Combinado a sobresalir, destacando en primer lugar Barranca x DK-7088 con 17.07Tn/há, de grano de Maíz Amarillo

\section{CONCLUSIONES}

Bajo las condiciones de clima y suelo en que se condujo el presente estudio, se dan las siguientes características:

1. De las principales características fenológicas de Maíz Amarillo Duro en valles e híbridos, destacan.

Longitud de Mazorca: Ica-Macacona con

$16.18 \mathrm{~cm} \mathrm{XB-8010} \mathrm{con} 16.61 \mathrm{~cm}$

Diámetro de Mazorca : Ica - Macacona con

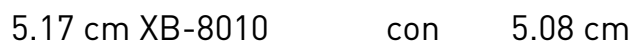

Número de Hileras x Mazorca: Ica-Macacona con $\quad 16.30$ AG-1596 con $\quad 17.10$

Húmero de Granos x hilera : Ica -

Macacona con 34.70 XB-8014 con $\quad 34.60$

Peso de grano (kg/parc): Barranca - La Paz

con $\quad 9.125 \mathrm{~kg} \mathrm{DK}-7088$ con $8.200 \mathrm{~kg}$

Peso de coronta (kg/parc): Barranca - La Paz con $1,650 \mathrm{~kg}$ XB-8018 con $1.500 \mathrm{~kg}$

Porcentaje grano (\%) : Trujillo - Moche con

$86.65 \%$ DK-7088 con $87.11 \%$

Peso 1,000 granos $(\mathrm{kg})$ : Cañete - Cerro Alegre con $0.60 \mathrm{~kg}$ XB-804 con $0.357 \mathrm{~kg}$

Peso x parcela gramo $(\mathrm{kg})$ : Barranca - La Paz con11.907 kg DK-7088 con $8.371 \mathrm{~kg}$

2. En Rendimiento Maíz Grano. Tn/há por Valles, destacan los híbridos: Departamento de Ica, Valle Ica - Macacona; DK-7088 con 14.923 Tn/há, XB-8016 con 14.900 Tn/há, Departamento de Lima. Valle Cañete - Cerro Alegre; XB-8018 con 12.492 Tn/há, AG-1596 con 12.374 Tn/há., Departamento de Lima. Valle Barranca - La Paz: DK-7088 con 17.073 Tn/há; XB-8018 con 15.005 Tn/há, Departamento La Libertad. Valle Trujillo Moche: XB-8016 con 12.820 Tn/há, DK-7088 con $12.654 \mathrm{Tn} /$ há, Departamento Lambayeque. Valle Chiclayo - Ferreñafe, DK-7088 con $10.136 \mathrm{Tn} /$ há, AG-1596 con 9.714 Tn/há, Departamento de Lambayeque. Valle Chiclayo - Motupe: XB-8018 con 14.50 Tn/há, XB-8014 con 14.345 Tn/há.

3. En Rendimiento Maíz Grano Tn/há destacan para:. Combinado Valle (V) x Híbrido (H): Barranca x DK-7088 con 17.070 Tn/há, sigue Barranca x XB-8018 con 15.00 Tn/há, Ica x DK7088 con 14.920 Tn/há, Ica - XB-8016 con 14.90 Tn/há, Valles (V): Barranca (Lima) con 14.250 Tn/ há, Ica (Ica) con 13.820 Tn/há, Híbridos (H): DK7088 con $13.420 \mathrm{Tn} / \mathrm{há}, \mathrm{XB}-8018$ con $12.670 \mathrm{Tn} /$ há; XB-8016 con 12.460 Tn/há.

\section{REFERENCIAS BIBLIOGRÁFICAS}

Alcántara, J., \& Quispe, K. (2017). Determinantes de la importación de maíz amarillo duro en el Perú, en los años 2007 - 2016. (Tesis de Licenciatura, Universidad Privada Antonio Guillermo Urrelo. Cajamarca, Perú). Recuperado de $53 \mathrm{http}: / /$ repositorio.upagu.edu.pe /handle/ UPAGU/699 [Consulta: 12 de enero del 2019].

Armijos E y Ruilova F. (2014.) Evaluación agronómica y adaptación de 12 híbridos comerciales y 4 híbridos experimentales de maíz (Zea mays L.) en 3 localidades, en las provincias de Loja y Santa Elena. Tesis para optar el título de Ingeniero Agrónomo. Cuenca-Ecuador. 185 p.

Banda (2017) Comparativo de rendimiento de 03 híbridos promisorios de maíz amarillo duro (Zea mays L.) y 5 testigos comerciales en el distrito de Batangrande - Santa clara. Lambayeque 2019. Castope z, A0. 2014. Comparativo de veintiséis híbridos de maíz amarillo duro (Zea mays l.) con fines forrajeros en Laredo, Trujillo. 
Chura J, \& Tejada J.. (2014). Behavior of yellow corn hybrids in town of La Molina, Perú. Idesia (Arica), 32(1), 113-118.

Diaz S. Comparativo de rendimiento de 03 híbridos promisorios de maíz amarillo duro (Zea mays L.) y 5 testigos comerciales en el distrito de Batangrande - Santa clara. Lambayeque 2019.

Espinoza, J. Fraccionamiento de nitrógeno en dos densidades de siembra de maíz amarillo duro (zea mays l.) en la localidad de la Molina.[ Tesis para optar el título de ingeniero agrónomo] Lima - Perú 2016.

Instituto Nacional de Innovación Agraria (INIA), Tecnología en Maíz - 2015 (http://www.inia.gob. pe/tecnologias/cultivos/132-cat-tecnologias/ cultivos/395- tecnologia-en-maiz).

MINAGRI. 2019. Serie estadística regional para el cultivo de maíz chala de los años 2015 al 2017. Disponible en http://frenteweb.minagri.gob.pe/ sisca/?mod=consulta_cult

Posada, C. (2018). Producción De Maíz Amarillo Duro No Cubre La Demanda Local. Cámara de Comercio De Lima. Recuperado de: https:// www.camaralima.org.pe/repositorioaps/0/0/ par/posada_841/posada_841_prod ucción de maíz amarillo duro no cubre la demanda local. pdf [Consulta: 09 de enero del 2019].

Quimi, D. 2015. Interacción genotipo-ambiente de híbridos triples experimentales de maíz (Zea mays L.), en dos zonas del litoral ecuatoriano. Tesis para optar el título de Ingeniero en Ciencias Agrarias. Quevedo - Los Ríos - Ecuador. 73 p.

Vasquez J. (2019)Adaptación de tres variedades de maíz amarillo (zea mays l.) para forraje en condiciones de la localidad de la Molina. [Tesis para optar el título de ingeniero agrónomo] Lima- Perú 2019. 